University of Nebraska - Lincoln

DigitalCommons@University of Nebraska - Lincoln

6-2001

\title{
Sharpening Low-Energy, Standard-Model Tests via Correlation Coefficients in Neutron $\boldsymbol{\beta}$ Decay
}

S. Gardner

University of Kentucky, gardner@pa.uky.edu

Chi Zhang

University of Nebraska-Lincoln, zhang.chi@unl.edu

Follow this and additional works at: https://digitalcommons.unl.edu/bioscifacpub

Gardner, S. and Zhang, Chi, "Sharpening Low-Energy, Standard-Model Tests via Correlation Coefficients in Neutron $\beta$ Decay" (2001). Faculty Publications in the Biological Sciences. 245.

https://digitalcommons.unl.edu/bioscifacpub/245

This Article is brought to you for free and open access by the Papers in the Biological Sciences at DigitalCommons@University of Nebraska - Lincoln. It has been accepted for inclusion in Faculty Publications in the Biological Sciences by an authorized administrator of DigitalCommons@University of Nebraska - Lincoln. 


\title{
Sharpening Low-Energy, Standard-Model Tests via Correlation Coefficients in Neutron $\beta$ Decay
}

\author{
S. Gardner* and C. Zhang \\ Department of Physics and Astronomy, University of Kentucky, Lexington, Kentucky 40506-0055
}

(Received 8 December 2000)

\begin{abstract}
The correlation coefficients $a, A$, and $B$ in neutron $\beta$ decay are proportional to the ratio of the axialvector-to-vector weak coupling constants, $g_{A} / g_{V}$, to leading recoil order. With the advent of the next generation of neutron-decay experiments, the recoil-order corrections to these expressions become experimentally accessible, admitting a plurality of standard model (SM) tests. The measurement of both $a$ and $A$, e.g., allows one to test the conserved-vector-current (CVC) hypothesis and to search for second-class currents (SCC) independently. The anticipated precision of these measurements suggests that the bounds on CVC violation and SCC from studies of nuclear $\beta$ decay can be qualitatively bettered.
\end{abstract}

Precision nuclear $\beta$-decay measurements have played an important role in the rise of the standard model (SM), giving strong credence to the conserved-vectorcurrent (CVC) hypothesis, as well as to the absence of second-class currents (SCC). We show that upcoming neutron-decay experiments can sharpen tests of the CVC hypothesis and of the absence of SCC significantly, eliminating assumptions inherent to the nuclear studies.

Searches for CVC violation and SCC in nuclear $\beta$ decay experiments have spanned decades of effort. We consider a CVC test originally suggested by Gell-Mann [1]: the strength of the "weak magnetism" term of the nucleon weak current ought to be given by the strength of the corresponding electromagnetic M1 transition. The SM test realized from such a comparison constrains a combination of the weak magnetism and induced tensor terms of the nucleon weak current. The induced tensor term is a "second class" current and thus is zero in the SM [2], save for isospin-violating effects engendered by the differing mass and charge of the $u$ and $d$ quarks. In tests of this sort, the CVC hypothesis is tested if SCC are assumed to be zero, or, alternatively, the nonexistence of SCC is tested if the CVC hypothesis is assumed to be valid.

Historically, the best constraints on the nonexistence of SCC and CVC violation are realized in the mass- 12 system [3,4]. The CVC hypothesis can be tested through the comparison of the spectral shape correction parameters $a_{\mp}$ measured in ${ }^{12} \mathrm{~B} \rightarrow{ }^{12} \mathrm{C}$ and ${ }^{12} \mathrm{~N} \rightarrow{ }^{12} \mathrm{C}$ transitions with the strength of the electromagnetic M1 transition from the analog state of ${ }^{12} \mathrm{C}$. This procedure yields a test of the CVC hypothesis at the 10\% level [3-5]. In order to realize a SCC test, the decays of spin-aligned ${ }^{12} \mathrm{~B}$ and ${ }^{12} \mathrm{~N}$ nuclei are studied. For purely aligned $1^{+} \rightarrow 0^{+}$transitions [6], the $e^{\bar{F}}$ angular distribution for ${ }^{12} \mathrm{~B}(-)$ and ${ }^{12} \mathrm{~N}(+)$ decay is given by [4]

$$
W_{\mp}\left(E_{e}, \theta, \mathcal{A}\right) \propto p_{e} E_{e}\left(E_{e}-E_{e}^{\max }\right)^{2}\left[1+\mathcal{A} \alpha_{\mp} P_{2}(\cos \theta)\right],
$$

where $p_{e}$ and $E_{e}$ are the momentum and energy of the electron (positron), $E_{e}^{\max }$ is the end point energy, $\theta$ is the angle between $\mathbf{p}_{\mathbf{e}}$ and the spin orientation axis, and $\mathcal{A}$ is the nuclear alignment. The difference $\alpha_{-}-\alpha_{+}$ is sensitive to the weak magnetism term as well as to the induced tensor term in the nucleon weak current. Unfortunately, it is also sensitive to the difference of the axial charges $\left(\Delta y \equiv y_{+}-y_{-}\right)$in the mirror transitions ${ }^{12} \mathrm{~B} \rightarrow{ }^{12} \mathrm{C}$ and ${ }^{12} \mathrm{~N} \rightarrow{ }^{12} \mathrm{C}$ - this potentiality has been included in only the most recent set of SCC tests $[7,8]$. Were $\Delta y=0$ and the experimental weak magnetism contribution determined from the M1 electromagnetic transition strength from the analog state of ${ }^{12} \mathrm{C}$ [9], as per the CVC hypothesis, Refs. [7] and [8] would yield $2 M f_{T} / f_{A}=0.12 \pm 0.05$ (stat) \pm 0.15 (syst) and $2 M f_{T} / f_{A}=0.04 \pm 0.16$ (stat) \pm 0.04 (syst), respectively. Note that $f_{T}$ and $f_{A}$ denote the induced-tensor and axial-vector coupling constants of the nucleon - the impulse approximation has been made in order to relate the nuclear and nucleon weak constants, note, e.g., Ref. [10]. This is consistent with the earlier result $2 M f_{T} / f_{A}=$ $-0.21 \pm 0.63$ [10]. Using $\Delta y=0.10 \pm 0.05$ [11], Refs. [7] and [8] determine that $2 M f_{T} / f_{A}=0.22 \pm$ 0.05 (stat) \pm 0.15 (syst) \pm 0.05 (theor) and $2 M f_{T} / f_{A}=$ $0.14 \pm 0.16$ (stat) \pm 0.04 (syst) \pm 0.05 (theor), yielding the combined constraint $0.01 \leq 2 M f_{T} / f_{A} \leq 0.34$ at 90\% C.L. [8]. This result suggests that $f_{T}$ is nonzero, with a value considerably in excess of SM expectations $[12,13]$. The inferred SCC contribution emerges from assuming the CVC hypothesis; alternatively, we can assert that SCC are identically zero in order to ascertain the quantitative validity of the CVC hypothesis. The uncertainties in the SCC determination are roughly 5\% of the value of the weak magnetism contribution, so that the CVC hypothesis is tested to this level. Note that an analogous test of SCC is possible in the mass- 8 system as well [14-16], yielding a second-class, induced-tensor nuclear form factor which is consistent with zero [14,15], albeit with an error rather larger than in the mass-12 
system. The mass- $8 \mathrm{CVC} / \mathrm{SCC}$ studies ought also to suffer a theoretical correction from the difference in the allowed axial matrix elements in the mirror ${ }^{8} \mathrm{Li} \rightarrow{ }^{8} \mathrm{Be}$ and ${ }^{8} \mathrm{~B} \rightarrow{ }^{8} \mathrm{Be}$ decays; in Refs. [14,15] this correction is set to zero.

We believe that a crisper test of the CVC hypothesis and of the nonexistence of SCC can be realized via the empirical determination of the correlation coefficients of neutron $\beta$ decay. Thus far, the especial focus of these experiments has been the determination of the CabibboKobayashi-Maskawa (CKM) matrix element $V_{u d}$. The latter is extracted from $g_{V}$, which is determined from the neutron-spin-electron-momentum correlation $A$ and the neutron lifetime $\tau_{n}$. The various determinations of $A$ do not agree [17]; a scale factor of 1.9 is assigned to the determination of $g_{A} / g_{V}$ from the measured values of $A$ by
Ref. [18]. These measurements were realized in reactor beam experiments; $A$ can also be measured using ultracold neutron sources - the systematic errors in such experiments are very different and would seem to be much smaller [19]. Nevertheless, the extracted value of $V_{u d}$, in concert with $V_{u s}$ from $K_{l 3}$ decays, tests the "squashed" unitarity relation $\left|V_{u d}\right|^{2}+\left|V_{u s}\right|^{2}+\left|V_{u b}\right|^{2}=1$ to better than $1 \%$. $V_{u d}$ may also be determined, indeed, more precisely, from the "superallowed" $0^{+} \rightarrow 0^{+}$decays in nuclei. In this case the empirical unitarity relation deviates from unity by $2.2 \sigma$; it is worth noting, however, that in this case the estimated theoretical errors dominate the presumed error bar [20].

Let us consider the correlation coefficients in neutron $\beta$ decay. The differential decay rate of a free neutron is given by [21]

$$
d^{3} \Gamma \propto E_{e}\left|\mathbf{p}_{\mathbf{e}}\right|\left(E_{e}^{\max }-E_{e}\right)^{2}\left[1+a \frac{\mathbf{p}_{\mathbf{e}} \cdot \mathbf{p}_{\nu}}{E_{e} E_{\nu}}+A \frac{\mathcal{P} \cdot \mathbf{p}_{\mathbf{e}}}{E_{e}}+B \frac{\mathcal{P} \cdot \mathbf{p}_{\nu}}{E_{\nu}}+D \frac{\mathcal{P} \cdot\left(\mathbf{p}_{\mathbf{e}} \times \mathbf{p}_{\nu}\right)}{E_{e} E_{\nu}}\right] d E_{e} d \Omega_{e} d \Omega_{\nu},
$$

where $\mathcal{P}$ denotes the neutron's polarization vector. The pseudo-T-odd coefficient $D$ is small [22] and can be neglected. Defining $\lambda \equiv\left|g_{A}\right| /\left|g_{V}\right|$ and neglecting terms of recoil order we have in the SM

$$
\begin{gathered}
a=\frac{1-\lambda^{2}}{1+3 \lambda^{2}} ; \quad A=2 \frac{\lambda(1-\lambda)}{1+3 \lambda^{2}}, \\
B=2 \frac{\lambda(1+\lambda)}{1+3 \lambda^{2}},
\end{gathered}
$$

implying that [23] $1+A-B-a=0$ and $a B-A-$ $A^{2}=0$. Currently [18]

$$
\begin{aligned}
& a=-0.102 \pm 0.005 ; \quad A=-0.1162 \pm 0.0013 \\
& B=0.983 \pm 0.004
\end{aligned}
$$

so that the relations implied by Eq. (2) are satisfied at the current level of precision - however, they do not hold once terms of recoil order are included in Eq. (2). The recoil-order terms are controlled by the dimensionless ratio of the electron energy to the neutron rest mass and thus are of $\mathcal{O}\left(10^{-3}\right)$, so that they impact $a$ and $A$ at the
$1 \%$ level. The correlation coefficient $B$ is much larger, so that the recoil-order terms become important only at the $0.1 \%$ level. Consequently we focus on what can be learned from $a$ and $A$. Recent experimental proposals suggest that $A$ and possibly $a$ can be measured to $0.2 \%$ or better $[19,24]$. We point out that additional standard model tests are possible once terms of recoil order become empirically accessible. In particular, one is sensitive to both the weak magnetism term $f_{2}$ as well as to the induced tensor term $g_{2}$ in the nucleon weak current. Indeed, independent tests of the CVC hypothesis and of the nonexistence of SCC are possible, as we now see.

The matrix element for polarized neutron $\beta$ decay in the $\mathrm{SM}$ is given by

$$
\mathcal{M}=\frac{G_{F}}{\sqrt{2}}\left\langle p\left|J^{\mu}(0)\right| \vec{n}\right\rangle \times\left[\bar{u}_{e}\left(p_{e}\right) \gamma_{\mu}\left(1+\gamma_{5}\right) u_{\nu}\left(p_{\nu}\right)\right] .
$$

We adopt the historic $\left(1+\gamma_{5}\right)$ sign convention in order to retain manifest consistency with earlier work [25-27]. Lorentz and translational invariance implies that the nucleon weak current $\left\langle p\left|J^{\mu}(0)\right| \vec{n}\right\rangle$ has six terms:

$$
\begin{aligned}
\left\langle p\left(p^{\prime}\right)\left|J^{\mu}(0)\right| \vec{n}(p, \mathbf{s})\right\rangle=\bar{u}_{p}\left(p^{\prime}\right)[ & f_{1}\left(q^{2}\right) \gamma^{\mu}-i \frac{f_{2}\left(q^{2}\right)}{M} \sigma^{\mu \nu} q_{\nu} \\
& \left.+\frac{f_{3}\left(q^{2}\right)}{M} q^{\mu}+g_{1}\left(q^{2}\right) \gamma^{\mu} \gamma_{5}-i \frac{g_{2}\left(q^{2}\right)}{M} \sigma^{\mu \nu} \gamma_{5} q_{\nu}+\frac{g_{3}\left(q^{2}\right)}{M} \gamma_{5} q^{\mu}\right] u_{n}(p, \mathbf{s}),
\end{aligned}
$$

where $\sigma^{\mu \nu}=\frac{i}{2}\left[\gamma^{\mu}, \gamma^{\nu}\right]$ and $q=p-p^{\prime}$. Note that $f_{1}(0)=g_{V}, \quad g_{1}(0)=-g_{A}=-f_{A} / G_{F}, \quad$ and $\quad g_{2}(0)=$ $-f_{T} M / G_{F}$, whereas $M$ and $M^{\prime}$ are the neutron and proton mass, respectively. The differential decay rate is given by

$$
\begin{aligned}
d^{3} \Gamma= & \frac{\left|G_{F}\right|^{2}}{2(2 \pi)^{5}} \frac{\left|\mathbf{p}_{\mathbf{e}}\right|\left|\mathbf{p}_{\nu}\right|}{M-E_{e}+\left|\mathbf{p}_{\mathbf{e}}\right| \cos \theta} \\
& \times\left[C_{1}+C_{2}\left(\mathcal{P} \cdot \mathbf{p}_{e}\right)+C_{3}\left(\mathcal{P} \cdot \mathbf{p}_{\nu}\right)\right. \\
& \left.\quad+C_{4} \mathcal{P} \cdot\left(\mathbf{p}_{\mathbf{e}} \times \mathbf{p}_{\nu}\right)\right] d E_{e} d \Omega_{e} d \Omega_{\nu},
\end{aligned}
$$


where the coefficients $C_{i}$ contain the form factors of Eq. (5) and are detailed in Ref. [25]. Note that $\theta$ is the angle between the electron and neutrino momenta in the neutron rest frame. Our particular interest is the recoil corrections to $a$ and $A$. Let us first consider the case in which the neutron is unpolarized. We have

$$
d^{2} \Gamma=\frac{2\left|G_{F}\right|^{2}\left|g_{\nu}\right|^{2}}{(2 \pi)^{4}} \frac{(M R)^{4} \beta x^{2}(1-x)^{2}}{(1-R x+R x \beta \cos \theta)^{3}}\left[C_{a}+C_{b} \beta \cos \theta\right] d E_{e} d \Omega_{e \nu},
$$

where

$$
R=\frac{E_{e}^{\max }}{M}=\frac{1}{2}\left(1+\epsilon-\eta^{2}\right) ; \quad x=\frac{E_{e}}{E_{e}^{\max }}, \quad \eta=\frac{M^{\prime}}{M} ; \quad \epsilon=\left(\frac{m_{e}}{M}\right)^{2},
$$

and $C_{a}+C_{b} \cos \theta=C_{1} /\left(2 M E_{\nu} E_{l}\left|g_{V}\right|^{2}\right)-C_{1}$ contains the electron-antineutrino correlation, $a$. Working in leading recoil order, including the phase-space contributions, we have

$$
d^{2} \Gamma=\frac{2\left|G_{F}\right|^{2}\left|g_{\nu}\right|^{2}}{(2 \pi)^{4}}(M R)^{4} \beta x^{2}(1-x)^{2}\left[\tilde{C}_{a}+\tilde{C}_{b} \beta \cos \theta+\tilde{C}_{c} \beta^{2} \cos ^{2} \theta\right] d E_{e} d \Omega_{e \nu} .
$$

Noting Eq. (1) we have $a=\tilde{C}_{b} /\left(\tilde{C}_{a}+\tilde{C}_{c} \beta^{2} \cos ^{2} \theta\right)$ and thus

$$
\begin{aligned}
a=\frac{1-\lambda^{2}}{1+3 \lambda^{2}}+\frac{1}{\left(1+3 \lambda^{2}\right)^{2}}\{ & \frac{\epsilon}{R x}\left[\left(1-\lambda^{2}\right)\left(1+2 \lambda+\lambda^{2}+2 \lambda \tilde{g}_{2}+4 \lambda \tilde{f}_{2}-2 \tilde{f}_{3}\right)\right] \\
& +4 R\left[\left(1+\lambda^{2}\right)\left[\lambda^{2}+\lambda+2 \lambda\left(\tilde{f}_{2}+\tilde{g}_{2}\right)\right]\right]-R x\left[3\left(1+3 \lambda^{2}\right)^{2}+8 \lambda\left(1+\lambda^{2}\right)\right. \\
& \left.\left.\times\left(1+2 \tilde{f}_{2}\right)+3\left(\lambda^{2}-1\right)^{2} \beta^{2} \cos ^{2} \theta\right]\right\}+\mathcal{O}\left(R^{2}, \epsilon\right),
\end{aligned}
$$

with $\tilde{f}_{2} \equiv f_{2}(0) / f_{1}(0), \tilde{f}_{3} \equiv f_{3}(0) / f_{1}(0)$, and $\tilde{g}_{2} \equiv g_{2}(0) / f_{1}(0)$. The momentum dependence of the form factors does not appear, as this effect first enters in next-to-leading recoil order. If $\tilde{f}_{3}=\tilde{g}_{2}=0$, this expression becomes that of Ref. [26]. Note, too, that it is also in agreement with Ref. [28].

The recoil correction to $A$ is determined from Eq. (6) by integrating over the neutrino variables. We find [25]

$$
d^{2} \Gamma=\frac{2\left|G_{F}\right|^{2}\left|g_{V}\right|^{2}}{(2 \pi)^{3}} \frac{\beta x^{2}(1-x)^{2}}{(1+\epsilon-2 R x)^{3}}\left[C_{a}^{\prime}+C_{b}^{\prime} \beta \mathcal{P} \cos \theta_{\mathcal{P}}\right] d E_{e} d\left(\cos \theta_{\mathcal{P}}\right),
$$

where $\theta_{\mathcal{P}}$ is the angle between the neutron's polarization vector and the electron momentum in the neutron rest frame. $C_{2}$ and $C_{3}$ give rise to $C_{b}^{\prime}$, whereas $C_{1}$ gives rise to $C_{a}^{\prime}$. Noting $A=C_{b}^{\prime} / C_{a}^{\prime}$, we have

$$
\begin{aligned}
A=\frac{2 \lambda(1-\lambda)}{1+3 \lambda^{2}}+\frac{1}{\left(1+3 \lambda^{2}\right)^{2}}\{ & \frac{\epsilon}{R x}\left[4 \lambda^{2}(1-\lambda)\left(1+\lambda+2 \tilde{f}_{2}\right)+4 \lambda(1-\lambda)\left(\lambda \tilde{g}_{2}-\tilde{f}_{3}\right)\right] \\
& +R\left[\frac{2}{3}\left[1+\lambda+2\left(\tilde{f}_{2}+\tilde{g}_{2}\right)\right]\left(3 \lambda^{2}+2 \lambda-1\right)\right] \\
& \left.+R x\left[\frac{2}{3}\left(1+\lambda+2 \tilde{f}_{2}\right)\left(1-5 \lambda-9 \lambda^{2}-3 \lambda^{3}\right)+\frac{4}{3} \tilde{g}_{2}\left(1+\lambda+3 \lambda^{2}+3 \lambda^{3}\right)\right]\right\} \\
& +\mathcal{O}\left(R^{2}, \epsilon\right) .
\end{aligned}
$$

If $\tilde{f}_{3}=\tilde{g}_{2}=0$, this expression becomes that of Refs. [26,29]. Our result is also in agreement with Ref. [28]. Our results are germane to hyperon decay as well; in this context either approximate expressions or the $E_{e}$-integrated asymmetry parameters are reported [30]. The recoil corrections to the correlation coefficients take the form $a_{0} R+a_{1} R x+a_{-1} \epsilon / R x$. The energy dependence of the three terms is distinct, although only two terms are empirically accessible as $\epsilon / R \sim$ $2.2 \times 10^{-4}$, whereas $R \sim 1.4 \times 10^{-3}$. Note that $x \in[\sqrt{\epsilon} / R, 1]$. Thus we have four independent empirical constraints, i.e., the $x^{0}$ and $x^{1}$ terms in $a$ and $A$, and three unknowns - namely, $\lambda, \tilde{f}_{2}$, and $\tilde{g}_{2}$. The system is overconstrained, so that we can infer the existence of physics beyond the SM, namely, the presence of non- $(V-A)$ currents [21], if the extracted coupling constants differ from SM bounds or if the values of the extracted couplings are not consistent with each other. Note that independent linear combinations of $\tilde{f}_{2}$ and $\tilde{g}_{2}$ appear in $a$ and $A$, so that, unlike the nuclear cases commonly studied, each coupling constant can be determined independently. Evaluating the recoil-order contributions to $a$ and $A$, using $\lambda=1.2670$ [18], $\tilde{g}_{2}=0$, and $\tilde{f}_{2}=\left(\kappa_{p}-\kappa_{n}\right) / 2=1.8529$, as per the CVC hypothesis, we find that the recoil corrections to $a$ are roughly a factor of 2 larger than those to $A$. By virtue of the allowed terms, $\lambda$ is determined to $0.030 \%$ and $0.022 \%$ by $0.1 \%$ measurements of $a$ and $A$, respectively. On statistical grounds, a precision measurement of $A$ would be the most efficacious in determining $\lambda$, whereas the determination of the coupling constants appearing in recoil order would seem to be better served with an $a$ measurement. $\tilde{f}_{2}$ and $\tilde{g}_{2}$ can be determined in a plurality of ways; let us illustrate. First, the $x^{1}$ and $x^{0}$ terms in $a$ can be determined to yield $\tilde{f}_{2}$ and $\tilde{f}_{2}+\tilde{g}_{2}$. $\lambda$ will be sufficiently precisely determined to have little impact on the errors in these parameters. Ignoring this source of error, we find a $0.1 \%$ measurement of $a$ yields a $2.5 \%$ measurement of $\tilde{f}_{2}$ from the $x^{1}$ term. This, in concert 
with the $x^{0}$ term from a $0.1 \%$ measurement of $a$, yields an uncertainty in $\tilde{g}_{2}$ of order $0.22 \lambda / 2$ - this is compatible with the errors quoted in the mass- 12 experiment with far fewer assumptions. Second, the $x^{1}$ dependence of the $a$ and $A$ terms can be determined - the former yields $\tilde{f}_{2}$, whereas the latter yields a combination of $\tilde{f}_{2}$ and $\tilde{g}_{2}$ [31]. Earlier determinations of $a$ were inferred from the recoil proton's spectral shape, see, e.g., Ref. [32], and were insensitive to the $x$ dependence of $a$; the newly proposed $a$ experiment [24] would be the first to measure $a$ as a function of $x$ [33]. The Fierz interference term, $b$ [21], which is zero in the SM can thus be bounded as well. Combining the earlier determination of $\tilde{f}_{2}$ with a $0.1 \%$ measurement of $A$ to determine $\tilde{g}_{2}$ from the $x^{1}$ term yields an uncertainty of $0.26 \lambda / 2$, commensurate with our earlier estimate. Although $0.1 \%$ measurements of $A$ seem quite feasible [34], measurements of $a$ to better than $1 \%$ may pose an especial challenge. Nevertheless, precision measurements of $a$ and $A$ are richly complementary. The measurement of both $a$ and $A$ permit crisp SM tests, namely, of SCC and the CVC hypothesis, not realizable in nuclear decays.

We thank M. S. Dewey and F. Wietfeldt for introducing us to the NIST " $a$ " experiment, and we gratefully acknowledge F. Wietfeldt, E. Adelberger, B. Filippone, B. Holstein, R. McKeown, K. Snover, and J. Tandean for additional helpful conversations and correspondence. We acknowledge the support of the DOE under Contract No. DE-FG02-96ER40989.

*Email address: gardner@pa.uky.edu

[1] M. Gell-Mann, Phys. Rev. 111, 362 (1958).

[2] S. Weinberg, Phys. Rev. 112, 1375 (1958).

[3] E. D. Commins and P. H. Bucksbaum, Weak Interactions of Leptons and Quarks (Cambridge University Press, Cambridge, 1983).

[4] L. Grenacs, Annu. Rev. Nucl. Part. Sci. 35, 455 (1985).

[5] L. De Braeckeleer, Phys. Rev. C 45, 1935 (1992).

[6] If we denote $p_{m}$ to be the fractional population in the $m=$ $1,0,-1$ magnetic substates, then the polarization $\mathcal{P} \equiv$ $p_{1}-p_{-1}$ and the alignment $\mathcal{A} \equiv p_{1}+p_{-1}-2 p_{0}$. A "purely aligned" target has $\mathcal{P}=0$.

[7] T. Minamisono et al., Phys. Rev. Lett. 80, 4132 (1998).

[8] K. Minamisono et al., Nucl. Phys. A663, 951 (2000).

[9] K. Koshigiri et al., Nucl. Phys. A319, 301 (1979); J. Phys. Soc. Jpn. Suppl. 55, 1014 (1986).

[10] Y. Masuda et al., Phys. Rev. Lett. 43, 1083 (1979), and references therein.

[11] K. Koshigiri et al., Nucl. Phys. A588, 165c (1995).

[12] J.F. Donoghue and B. R. Holstein, Phys. Rev. D 25, 206 (1982).

[13] H. Shiomi, Nucl. Phys. A603, 281 (1996).

[14] L. De Braeckeleer et al., Phys. Rev. C 51, 2778 (1995).

[15] K. Snover et al., in Proceedings of the International Symposium of Nuclear Electroweak Spectroscopy (NEWS99), Osaka, Japan, 1999 (World Scientific, Singapore, to be published).
[16] R.E. Tribble and G. T. Garvey, Phys. Rev. C 12, 967 (1975); R. D. McKeown, G. T. Garvey, and C. A. Gagliardi, Phys. Rev. C 22, 738 (1980); M. Beck et al., in Intersections Between Particle and Nuclear Physics, edited by T. W. Donnelly, AIP Conf. Proc. No. 412 (AIP Press, New York, 1997), p. 416.

[17] H. Abele et al., Phys. Lett. B 407, 212 (1997); P. Liaud et al., Nucl. Phys. A612, 53 (1997); B. G. Erozolimsky et al., Phys. Lett. B 412, 240 (1997); P. Bopp et al., Phys. Rev. Lett. 56, 919 (1986).

[18] Particle Data Group, D. E. Groom et al., Eur. Phys. J. C 15, 1 (2000).

[19] A Proposal for an Accurate Measurement of the Neutron Spin-Electron Angular Correlation in Polarized Neutron Beta Decay with Ultra-Cold Neutrons, T. Bowles and A. R. Young, co-Principal Investigators.

[20] I. S. Towner and J. C. Hardy, nucl-th/9809087.

[21] J. D. Jackson, S. B. Treiman, and H. W. Wyld, Jr., Phys. Rev. 106, 517 (1957); Nucl. Phys. 4, 206 (1957).

[22] emiT Collaboration, L. J. Lising et al., Phys. Rev. C 62, 055501 (2000).

[23] Yu. A. Mostovoi and A. I. Frank, JETP Lett. 24, 38 (1976).

[24] F. Wietfeldt et al., in Proceedings of the Fundamental Physics with Pulsed Neutron Sources Conference, Durham, NC, 2000 (to be published).

[25] D. R. Harrington, Phys. Rev. 120, 1482 (1960). Minor errors in the $C_{i}$ impact only the $f_{3}$ contribution to $A$ and $a$ in leading recoil order. The expressions reported for $C_{a}^{\prime}$ and $C_{b}^{\prime}$ and consequently for the recoil correction to $A$ are inconsistent with ours.

[26] S. M. Bilen'kii et al., Sov. Phys. JETP 37, 1241 (1960).

[27] I. Bender, V. Linke, and H. J. Rothe, Z. Phys. 212, 190 (1968).

[28] B. R. Holstein, Rev. Mod. Phys. 46, 789 (1974); 48, 673(E) (1976). Effecting the correspondence $a=f_{1}$, $b=\sqrt{3}\left(f_{1}+2 f_{2}\right), c=\sqrt{3} g_{1}, d=2 \sqrt{3} g_{2}, e=2 f_{3}$, and $h=2 \sqrt{3} g_{3}$, we find that the $f_{i}(E)$ of Eq. (51) are given by $f_{1}(E)+\left(f_{1}^{2}-g_{1}^{2}\right)[R x-\epsilon /(R x)]=\tilde{C}_{a}$, $f_{2}(E)=\tilde{C}_{b}$, and $f_{3}(E)=\tilde{C}_{c}$. The corrected $F_{i}$ of Eq. (52) are consistent with our $C_{a}^{\prime}$ and $C_{b}^{\prime}$ once the recoil-order terms from the phase-space factor $(1+\epsilon-2 R x)^{-3}$ are incorporated.

[29] D. H. Wilkinson, Nucl. Phys. A377, 474 (1982).

[30] V. Linke, Nucl. Phys. B12, 669 (1969); J. M. Watson and R. Winston, Phys. Rev. 181, 1907 (1969). Comparing Eq. (9) with the approximate treatment of the latter we find that the terms involving $\tilde{f}_{2}$ and $\tilde{g}_{2}$ in $R$ and $R x$ are identical, whereas the others differ. See also P. H. Frampton and W. K. Tung, Phys. Rev. D 3, 1114 (1971); Y. Yokoo, S. Suzuki, and M. Morita, Prog. Theor. Phys. 50, 1894 (1973). Note that Ref. [27] treats the unpolarized case; our $C_{a}^{\prime}$ can be recovered from their expressions.

[31] Reference [28] notes, at the bottom of p. 805, that the $b$ and $d$ terms can be determined independently from the energy dependence of the electron-antineutrino correlation and polarization asymmetry in an analog decay, such as ${ }^{19} \mathrm{Ne} \rightarrow{ }^{19} \mathrm{~F}$ or $n \rightarrow p$.

[32] C. Stratowa, R. Dobrozemsky, and P. Weinzierl, Phys. Rev. D 18, 3970 (1978).

[33] F. Wietfeldt (private communication).

[34] B. Filippone (private communication). 